\title{
A Model Driven Ontology-based Architecture for Supporting the Quality of Services in Pervasive Telemedicine Applications
}

\author{
Ebrahim Nageba \\ MTIC - EA4171 \\ INSA de Lyon, Université de Lyon \\ Bron, France \\ ebrahim.nageba@insa-lyon.fr
}

\author{
Jocelyne Fayn \\ INSERM, MTIC - EA4171 \\ INSA de Lyon, Université de Lyon \\ Bron, France \\ jocelyne.fayn@insa-lyon.fr
}

\author{
Paul Rubel \\ MTIC - EA4171 \\ INSA de Lyon, Université de Lyon \\ Bron, France \\ paul.rubel@insa-lyon.fr
}

\begin{abstract}
Advanced telemedicine systems based on ubiquitous computing need to be designed in order to deliver high quality of services at anytime and anywhere. The optimal management of the telemedical assistance process in emergency situations, taking into consideration different communication and material resources availability, is still a major problem in telemedicine. In this paper we propose an ontology-based architecture model enabling an intelligent pervasive telemedicine tasks management. The aim of this methodological approach is to optimize the messages exchange between the different actors who are geographically located in different environments, to assure high level of quality of services delivered by the healthcare providers and consequently enable a more rapid and reliable telemedical assistance, especially in case of emergency scenarios.
\end{abstract}

Keywords: telemedicine; ubiquitous computing; quality of service; context management; ontology.

\section{INTRODUCTION}

Telemedicine applications based on pervasive computing require automated and continuous services, and proactive real-time collaborations among devices, software agents and geographically distributed actors in dynamic, heterogeneous environments [1]. A common problem among the different telemedical applications is to ensure the quality of service in terms of information and communication between the different actors. In particular, telemedicine systems should include a self adaptative management of the various tasks to be performed according to the different contexts of use. However, the existing telemedicine systems do not pay enough attention to the quality level of their offered services nor offer adequate management of material and communication resources, distributed in various geographic locations such as hospitals and healthcare centers. Also bandwidth limitations and end-to-end communication delays should be taken into account. In addition, telemedicine systems architectures should be interoperable to allow and facilitate data exchange. The development of telemedicine solutions that are open to the multiplicity of the offered services, to the functional diversity of the systems and the user needs heterogeneity requires designing information and communication models which grant for every actor an optimal use of available services and resources.

The telemedicine scenarios involving pervasive computing applications are quite diverse. The most popular ones from the literature are for example home care and monitoring [2] [3] [4], assistance for healthcare professionals [5] [6] and Tele-expertise in cardiology [7] [8].

Recently, ontologies have become a popular instrument within the ambient intelligence knowledge engineering community for defining flexible, scalable, personalizable and open models of concepts and interrelations [9]. OWL (Ontology Web Language) has deserved much support both from the scientific community and from the World Wide World Consortium (W3C) these last years [10]. It is an expressive ontology language resulting in RDF (Resource Description Framework) type description, and has the capacity of supporting semantic interoperability to exchange and share knowledge between different systems in various domains and of enabling automated reasoning. Thanks to XML and OWL, it becomes easy to align different ontologies structures. The existence of foundational ontologies libraries should facilitate the development of ontology-based systems [11] [12] [13]. Furthermore, ontologies can provide a uniform way for specifying the context model's core concepts, sub-concepts and facts, and consequently enable contextual knowledge sharing and reuse in ubiquitous systems.

Context modeling and contextual situations management in telemedicine, particularly in emergency scenarios, should be considered for ensuring the provision of the right information at the right time at the right place. Ontologies have already been used for context modelling, representation and management in pervasive environments, and for services discovery and semantic interoperability among ubiquitous computing devices [14] [15]. Ontology-based context modelling approaches provide a set of ontological concepts to characterize entities such as a person, a place or several other kinds of objects within their context [16]. A context model survey is presented in [17]. Ontology Definition Metamodel (ODM) [18], specified by the OMG (Object 
Management Group), enables ontology modelling through the use of UML-based tools. Other approaches based on MDA (Model Driven Architecture) have been applied for context ontology modelling [19].

In the eHealth and telemedicine domains, ontologies are being used to propose context models for healthcare monitoring and home care [20] [21]. These models are based on predefined protocols to collect medical data which are used to automatically monitor the patient status and to detect possible alarm situations. Other research works highlighted the relevance of using ontologies to solve semantic interoperability problems in telemedicine applications [22]. However, architecture solutions able to dynamically support services and resources management in pervasive telemedicine applications have never been proposed. Therefore, the design of a model driven ontology-based architecture for supporting the knowledge management of resources availability and capability, enabling a better quality of ubiquitous telemedical services in different scenarios, especially in case of emergency, is still a challenge and emerging research and development area [23] [24]. .

The aim of this paper is to present an approach for building a knowledge-based system architecture for optimising the use of resources and the messages exchange among different actors, in order to enhance the quality of services in telemedicine applications. The core element of the system is an ontology-based model for an intelligent management of the different types of available communication and material resources. The final objective is to provide a more rapid and reliable medical assistance in telemedicine emergency scenarios.

In the next section, we detail two main telemedicine scenarios which are Tele-assistance and Tele-expertise in cardiology. In section 3, we briefly present the main components of the system architecture. Then, we describe the telemedicine tasks management process in section 4 . In the last section we give a detailed presentation of an ontology model representing the main concepts and their relations, and we detail some aspects of the ontology realization and implementation.

\section{TELEMEDICINE APPLICATION SCENARIO}

\section{A. Telemedicine Scenario Examples}

A typical telemedicine application scenario in pervasive environment concerns medical assistance in critical geographic or isolated areas, such as high mountains resorts. Let us suppose that a person has an accident or a heart attack while skiing or staying in a high mountains resort. The victim needs immediate assistance and healthcare provided by the rescue team members or by emergency physicians who are in geographically distributed locations. The healthcare providers need to know where the patient should be admitted according to different contextual factors such as the patient's clinical status, his social conditions, the hospital's location, etc., taking into account the availability and the capability of heterogeneous resources. An ideal pervasive information system should propose services according to the emergency physician profile and the context of care. Depending on the geographic location and the available material and human resources, the system will possibly have to infer several recipient addresses and to set up different messages that shall be addressed to one or more of these recipients. An effective collaboration framework is needed to save the victim. To start this collaboration, communication devices such as a cellular phone and pocket PCs with wireless connectivity can be used so that the user can access to the system anywhere and anytime. According to the actor profile and context, the system should offer several tasks to be performed in an intelligent manner in order to provide the highest level of quality of service. The offered tasks may be for instance Teleassistance for patient orientation or Electronic Health Record (EHR) access.

The rescue team members should be able to access the system to find an hospital or a healthcare center which has an available place and has the required human and material resources and competence to perform the required treatments to the patient, including specific or particular ones. Thus, three important factors should be taken into consideration by the solutions inferring process. Firstly, the location of the hospital or of the healthcare center to which the patient could be oriented, and secondly, the availability of the required logistical and material resources such as beds, surgery or intensive care units, etc. The third factor is the center's competence, which means the center's capability to perform the needed treatment. For these reasons, the system should infer several possible destinations depending on these three previous factors. Consequently, a sequence of solutions inferred by the system should be sent to the requesting actor so that he can finally choose the one that best fits his situation and context.

The possible destinations could be a general medical center, an intensive care unit or a specialized hospital. The messages exchanged by the system will be encapsulated in the XML format. The messages could be informative, such as a message to ask the intensive care unit to be ready to receive the patient, or advice request messages, for immediate drug administration for instance, which require a rapid response. The XML messages can include different types of data (i.e., patient's personal data, medical data such as blood pressure, symptoms descriptions, biosignals like an ECG, and eventually a list of drugs or a digital picture of a wound).

There are also other types of scenarios such as Teleexpertise in the cardiology domain between an emergency physician and a remote cardiologist, self care situations, follow up of patients at home, and follow up of rural populations or elderly in nursing homes. All these scenarios require models that shall enable an intelligent management of the services, resources and exchanged messages among the different actors to increase the quality of services delivered by the healthcare providers.

\section{B. Use Cases}

Starting from the user needs and the telemedicine system requirements, several use cases can be described according to 


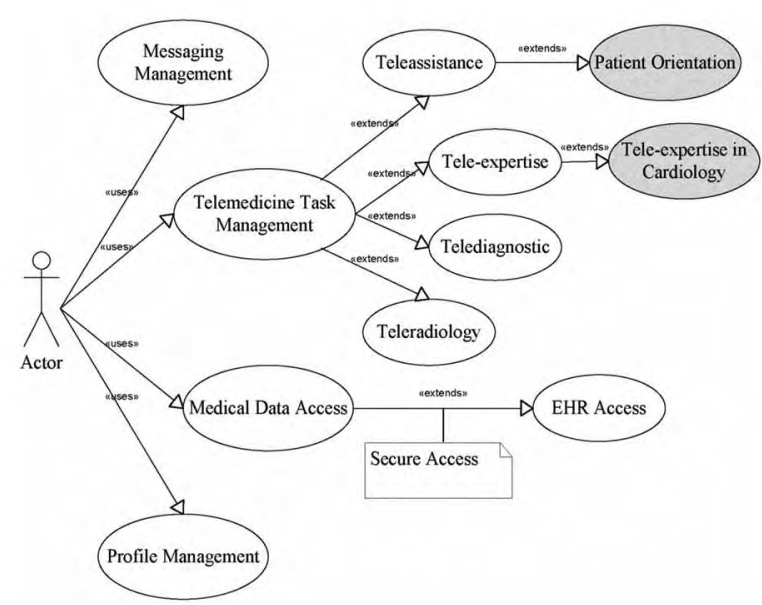

Figure 1. General use cases UML diagram

the application scenarios. Figure 1 displays a simplified model of the system's four main use cases represented according to the UML formalism:

- Telemedicine tasks management.

- Actor's profile management.

- Medical data and information access.

- Messaging management.

The most complex use case is the Telemedicine Tasks management. We define a Telemedicine Task as a set of processes (such as the use of generic and business services, the set up and transmission of a message ...) performed by a medical information system to provide the user with medical data and information that are relevant to his request.

The final objective of the tasks management in telemedicine applications is to provide the actors at the remote site with appropriate, rapid, reliable, and well documented telemedical assistance.

\section{SYSTEM COMPONENTS PRESENTATION}

Given the diversity of the scenarios mentioned in the previous section, the necessity of scalability and the complexity of the tasks, we designed a solution based on an ontology to support the management of the different tasks that shall be inferred by the telemedicine system, and XML to describe and format the messages that will be exchanged between the different actors. Figure 2 displays the general schema representing the main components of the proposed system. We provide hereafter a brief explanation of these main components which are the Task Management Server, a Communication Server and a Knowledge Base.

\section{A. Telemedicine Tasks Management Server}

The Tasks Management Server (TMS) is based on the W3C SPARQL Query Language for RDF [25]. SPARQL permits to retrieve data from the ontology through the query

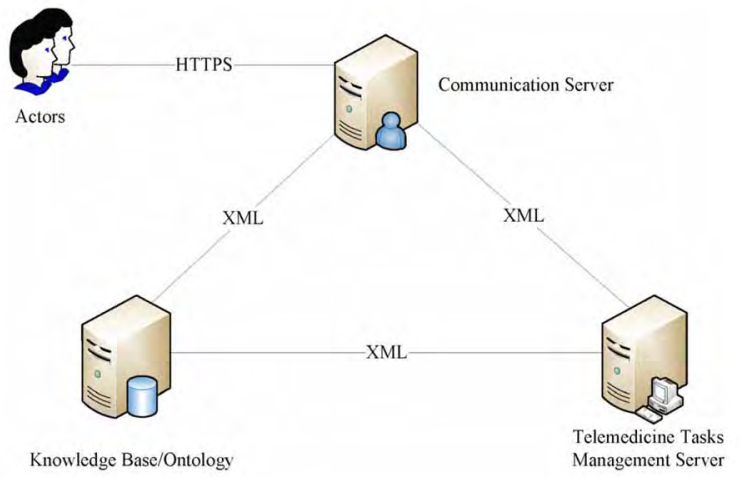

Figure 2. System architecture components graphic representation

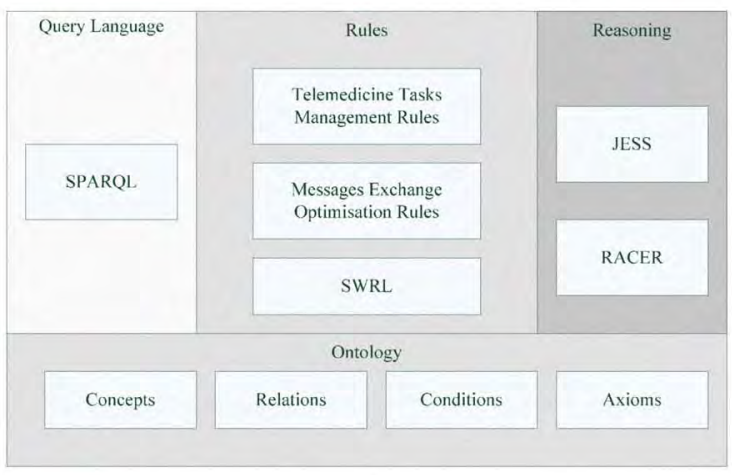

Figure 3. Knowledge base architecture components

forms SELECT, CONSTRUCT, ASK and DESCRIBE. It returns the query results in RDF or XML. Thus, the main role of the TMS is to link the actors with the tasks that they can operate, and the selected task with the required data. In addition, it shall set up XML messages encapsulating different types of medical data, viz heart rate frequency, systolic and diastolic blood pressure, body temperature, etc.

\section{B. Communication Server}

The communication server shall manage operations such as identification, authentification and the services that the user will be allowed to operate according to his profile. It shall also manage the exchange of XML messages issued by the Task Management Server by taking into account the emergency of delivery. For example the messages sent by an emergency physician must be assigned to a high priority. The communication server shall thus perform three major processes:

- Prioritize the messages coming from the Task Management Server according to the sender and receiver actors' priority.

- Stratify the messages in queues according to their priorities.

- Apply some transportation rules, such as, "send the first message in the high priority message queue; if 
there is no message in the high priority queue, send the first message in the medium priority message queue".

\section{Knowledge Base}

The core element of the architecture is the knowledge base. Figure 3 depicts its main components which are:

- An ontology for the telemedicine tasks concepts and concepts interrelations representation.

- A set of rules to be applied for decision making.

- The reasoning.

- A query language.

1) Telemedicine tasks ontology

The purpose of the ontology is to support the management of the messages exchange between the different actors of the telemedicine system (healthcare professionals, patients, software agents, intelligent devices ...). In other words, this ontology-based system shall offer solutions that are controlled according to a set of rules applied on or inferred from the knowledge represented by the ontology. Furthermore, using ontologies will ease the process of analysing knowledge by creating specific rules and profiles. The main role of the ontology is to capture knowledge about the resources and actors in the telemedicine domain, by describing the different concepts and their interrelations used by the tasks.

The ontology we have designed is composed of two layers. The first layer contains the description of the general concepts concerning the telemedicine services (such as Actor, Resource, Location ...) and the interrelations between these concepts. The second layer is the domain specialisation layer representing the different objects such as Specialist, PDA, and Tele-expertise.... The domain specific ontologies in each sub-domain (i.e. Hospital, Home ...) can be dynamically plugged into the upper ontology when the environment is changed.

\section{2) Rules and reasoning}

One of the key features of ontologies is that they can be processed by a Reasoner which supports the decision-making process and provides the knowledge base with the capacity of reasoning by applying defined rules. Various existing logic reasoning mechanisms can be exploited to deduce decisions that shall support the tasks management in telemedicine applications. These decisions shall optimise message exchange and transportation, and the use of resources. Additionally, the decisions shall provide solutions to societal problems such as the patient orientation in emergency cases. Using ontologies and an appropriate Reasoner allows to automatically infer the ontology class hierarchy and easies consistency checking. Rules can be defined in SWRL (Semantic Web Rule Language) [26] which is based on a combination of the OWL-DL (Description-Logic) and OWL-Lite sublanguages of OWL web ontology languages. The rules will be applied by a Reasoner like Jess (Java Expert System Shell) [27] for decision making.

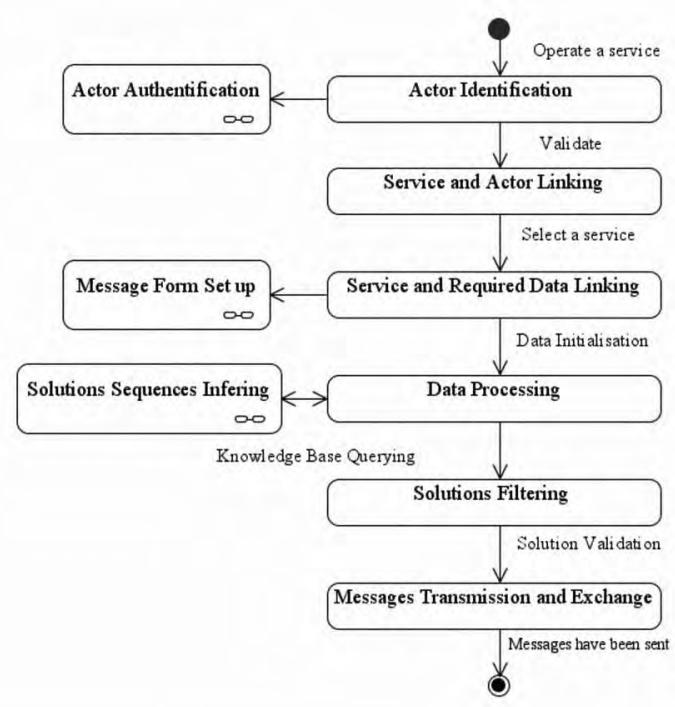

Figure 4. UML Machine States diagram representing the telemedicine task management process.

A typical rule for patient orientation decision making could be: "if the requested service is tele-assistance for patient orientation (Service) and if the patient needs coronary angioplasty and stenting (Resource) then propose to the emergency physician (Actor) the closest hospital qualified to perform this treatment".

\section{3) Query language}

To express queries across the different data sources, whether the data are stored in RDF or transformed in RDF via middleware, we use the SPARQL language. SPARQL contains capabilities for querying both required and optional graph patterns. The queries results are themselves a set of RDF graphs.

\section{TElemedicine TASKs ManagemENT PROCESS}

Three principal states types can be distinguished when the user interacts with an information system. The first one is the sending of a message from the actor towards the system, the second is a change in the system state and the third is the resending of a message from the system towards the actor. Figure 4 shows the UML machine states diagram representing the states sequences which describe the system behaviour with the transitions associated to every state. The main steps of the telemedicine task management process are described as follows:

\section{1) Actor identification and authentification}

Actor identification and authentification can be viewed as major legal requirements for the use of telemedicine applications from a legal point of view. Therefore, all actors are obliged to accept the use of some certificates, established by an official governmental organisation, to fulfil the legal requirements. 


\section{2) Actor/Services linking}

The relation actor/service has been defined in the designed ontology as (Actor Operates Service). According to the actor's profile some services have been defined to be operational (e.g. Cardiologist Operates Tele-expertise in Cardiology). Therefore, to restrain the operating of the service by the different types of actors, some restrictions have been added on the classes. We provide more details about the concepts and relations in the ontology realisation and implementation section.

\section{3) Service and required data linking}

We have defined the relation between the service and the data as (Service Requires Data). Therefore, once the actor selects a service to operate, the system links the selected service with the required data and requests the actors to initialize (enter) some of the required data through a message form. The ontology links all services to their related data. For example, the task "Tele-expertise In Cardiology" needs data such as patient personal data (i.e. name, sex, date of birth ...), medical data (i.e. ECG, Blood pressure ...). Thus, these data should be initialized by the requestor and sent to the recipient who will make a diagnosis based on the sent data. In another type of tasks like "Tele-assistance", other types of data can be initialized such as data about the pathology, data about the required treatment type, etc.

\section{4) Data processing and solution inferring}

Depending on the selected service, the available human, material and communication resources and the data initialized by the actor, the system infers a sequence of solutions by querying the ontology and applying defined rules on the queries results. For example, in the Teleassistance scenario for patient orientation in case of an accident or in an emergency situation, the query should be restricted on the type of required treatment. Based on these restrictions the query results should provide the destination or the hospital which has the material resources needed for the specific treatment type. An example of SPARQL query will be provided in section $\mathrm{V}$.

\section{5) Solutions filtering}

The inferred solutions should be classified and filtered according to some factors or criteria such as location, technical properties of communication devices of sender and receiver actors and the availability of some specific logistical or material resources. The filtering process can be performed on two levels. The first one is carried out through the ontology querying by including the FILTER pattern in the query syntax. The second filtering level is performed by the Reasoner by applying predefined rules.

\section{6) Messages transmission and exchange}

The system sends a set of messages to the concerned recipients according to the solution needed by the actor. As we mentioned above, the communication server performs this process by applying the defined communication rules to optimise the message exchange and to ensure the message reception by the recipient actor.

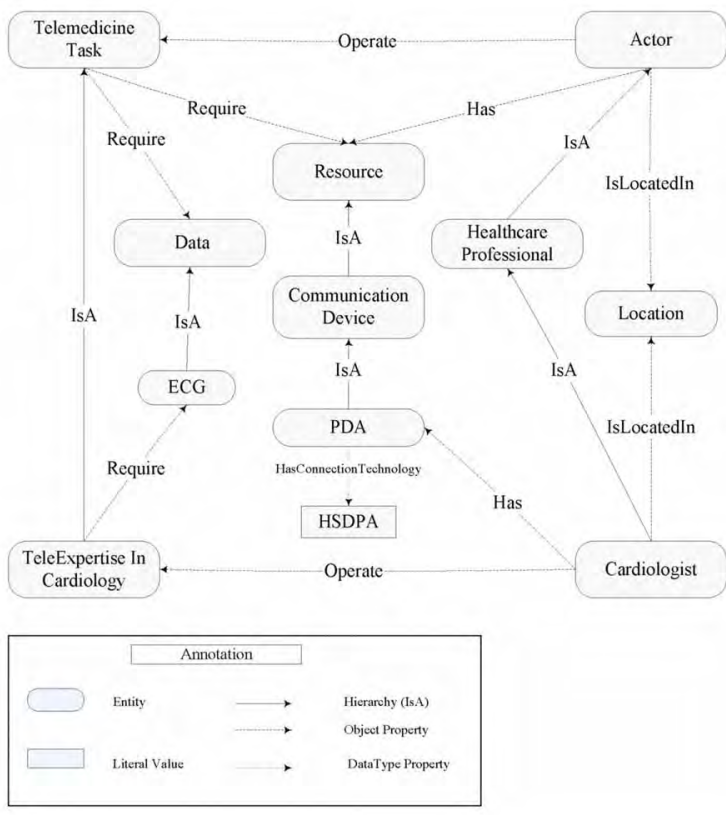

Figure 5. Main concepts and interrelations in the ontology-based telemedicine task model

\section{ONTOLOGY REALIZATION AND IMPLEMENTATION}

An ontology includes a set of entities and logic statements that specify what each entity means and how they are related to each other. In our ontology we have defined 5 main entities: Actor, Resource, Location, Data, and Telemedicine Task. The Actor entity represents classes of individuals such as healthcare professional, patient, institution (healthcare organisations). An actor has the following attributes: ID, name, general domain, speciality, location, etc. The Resources are the available material and communication resources. Their properties are: type of resource, brand description, communication technology, total memory, bandwidth, quantity, etc. The telemedicine tasks are classified in different subclasses according to the medical domain such as (cardiology, neurology, diabetes, cancer, etc). The Data class includes subclasses like Biosignals, Images, Textual data, etc.

To represent our ontology we have used the OWL-DL formalism. Figure 5 displays an extract of our ontology graph represented according to OWL-DL, showing some of the entities described above as well as their interrelations. In OWL-DL, there are two main types of properties that can be defined. The first type is Object properties. For example actor HAS resource, actor OPERATE service, service REQUIRE resource, service REQUIRE data. The second type of properties is Datatype properties. For example, Healthcare Professional HAS speciality. Individuals represent objects in the domain in which we are interested. For instance, emergency physician is an individual from the class health care professional. Properties link two individuals together. For example, the property OPERATE might link 
the individual emergency physician (Actor) with the individual Tele-expertise (Service). Also the property HAS might link the individual general practitioner X (Actor) to the individual PDA (Resource). Properties link individuals from the domain to individuals from the range. To illustrate, in the Telemedicine Task ontology, the property HAS links individuals belonging to the class Actor to individuals belonging to the class Resources. In this case, the HAS property domain is class Actor and the range is class Resources. This means that individuals that are used 'on the left hand side' of the HAS property will be inferred to be members of the class Actor. Any individual that is used 'on the right hand side' of the HAS property will be inferred to be members of the class Resources.

To edit the ontology we have used Protégé-OWL [28], a knowledge acquisition tool that facilitates the description of the concepts. To test the ontology consistency and class hierarchy we have used Reasoner Racer [29]. One of the main services offered by a Reasoner is to test whether or not one class is a subclass of another class. By performing such tests on all of the classes of the telemedicine tasks ontology it is thus possible to infer the ontology class hierarchy.

Based on the description of the necessary conditions and necessary and sufficient conditions of the different classes, the Reasoner checks whether or not all classes have at least one instance. The Reasoner then checks the conditions defined for each class to determine whether an individual satisfies the restrictions to be a member of a class. For example, for an individual $\mathrm{X}$ to be a member of the class Actor, it should at least operate one service and have one or more resources.

Figure 6 displays an extract from the RDF source code generated by Protégé describing the telemedicine task instance "EmergencyPatientOrientation" which is a subclass of "TeleAssistance". It shows the data that should be initialized to perform the patient orientation task: patient personal data, required treatment type, pathology and required resource.

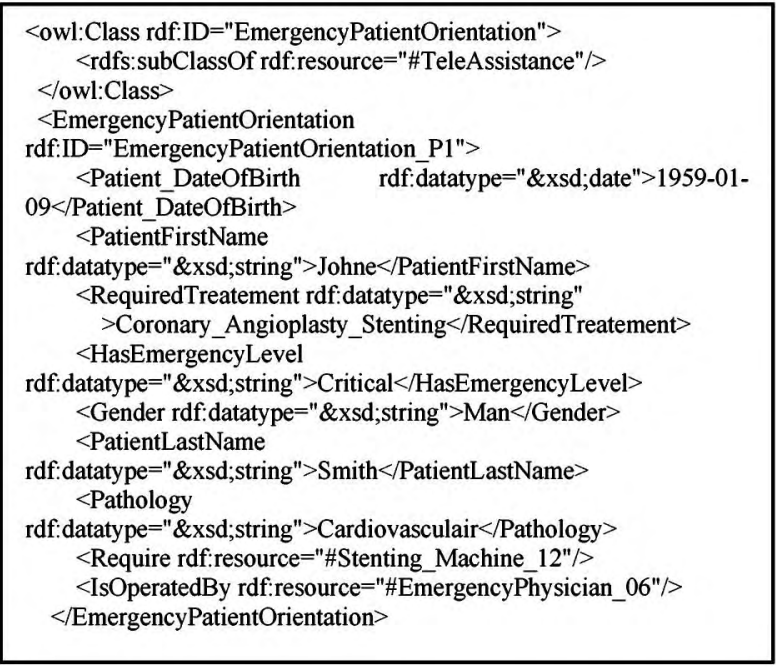

Figure 6. Extract of RDF source code generated by Protégé-OWL

Figure 7 shows an example of SPARQL query which shall return a set of actors, the telemedicine services they can use, the communication devices which are at their disposal, and their locations. We have added the modifier FILTER to limit the solutions so that we obtain only the actors who have communication devices having connection technology "HSPDA". The query results are shown in figure 8.

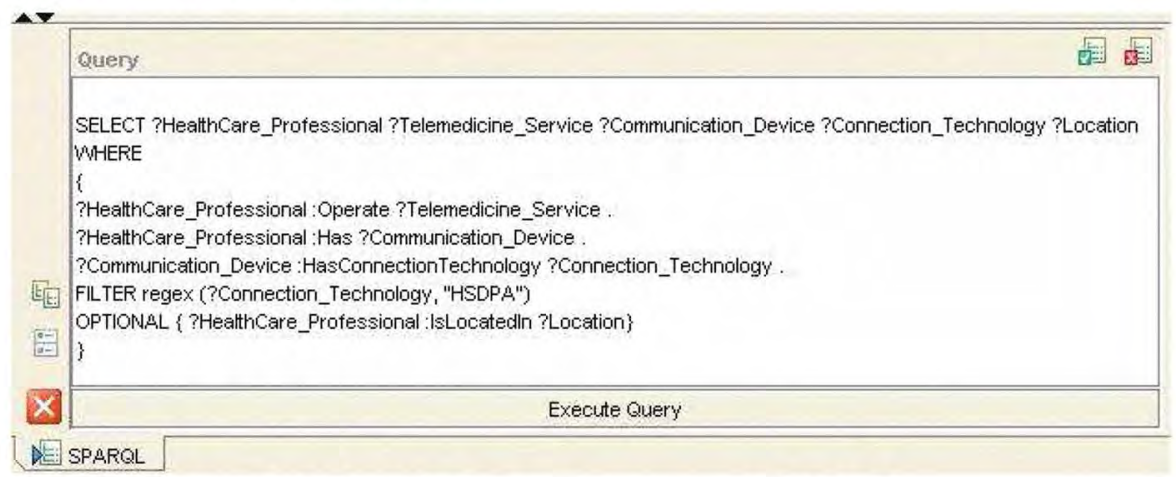

Figure 7. Telemedicine Tasks ontology query example in SPARQL

\begin{tabular}{|c|c|c|c|c|}
\hline \multicolumn{5}{|l|}{ Results } \\
\hline HeathCare_Professional & Telemedicine_Service & Communication_Device & Connection_Technology & Location \\
\hline EmergencyPhysician_06 & - EmergencyPatientorientation_P1 & HiTc_02 & HSDPA & Location_15 \\
\hline Cardiologist_01 & TeleExpertiselnCardiology_06 & Hitc_01 & HSDPA & - Hospital_Cardiologique_Lyon \\
\hline EmergencyPhysician_02 & EmergencyPatientOrientation_P5 & - iPhone_03 & HSDPA & Location_11 \\
\hline Cardiologist_06 & TeleExpertiselnCardiology_01 & PDA_06 & HSDPA & - HealthCareCenter_06 \\
\hline
\end{tabular}

Figure 8. Example of results obtained in answer to the SPARQL query of Fig. 7 


\section{DISCUSSION AND CONCLUSION}

The diversity and the specificities of the applications make the telemedicine tasks management a complex process. To master this complexity we have designed an ontology-based telemedicine task management system architecture that is composed of a Task management Server, a Communication Server and an ontology to represent the concepts such as Actor, Resource, Service and Medical Data, and the interrelations among these concepts. The ontology has been defined and edited by OWL-DL and implemented by means of the Protégé technology. Class hierarchy and ontology consistency have been checked thanks to the Racer Reasoner. The setting-up of a first demonstrator has shown that the proposed ontology-based telemedicine system architecture facilitates the design of complex telemedical assistance processes and the management of telemedicine messages exchange and thus should contribute to the enhancement of the quality of pervasive telemedical services. In contrast to others solutions that were proposed for the support of pre-defined telemedical scenarios such as the continuity of care at home of patients with chronic diseases, the architecture design we proposed in this paper aims to be generic enough for also being compliant with ubiquitous medical assistance in pervasive environment. The example of emergency scenario in high mountains or isolated areas we presented as an illustration of societal requirements is all the more relevant that it also affects young people with unknown prior health problems. Numerous studies have reported about cases of cardiac deaths among sportsmen and active citizens, while others have demonstrated the relationship between mortality and the time delay to an appropriate treatment of the heart diseases [30]. The results of the evaluation that has been performed by our group within the framework of the European EPI-MEDICS project [7, 31] has shown that thanks to the development of a Personal ECG Monitor (PEM) that any citizen can easily use anywhere at any time to self-record the electrical activity of his heart, one could save lives by automatically calling an emergency center in case of acute ischemia or life threatening arrhythmia. Another finding was that $10 \%$ of the patients involved in an experimental study of self-care presented new arrhythmia events that were never diagnosed before. The early and ubiquitous detection of malignant cardiac events, as soon as the first symptoms occur, is therefore easily affordable for everybody. The challenge is thus at present to provide the healthcare stakeholders with pervasive services allowing to reduce the time of patient's admission to the nearest and more appropriate medical center, taking into account in addition the ambient and context-aware information both specific to the patient's clinical status and lifestyle and to the offered possibilities in function of the geographical situation where the event occurred. The local healthcare regulation rules, the communication facilities, and the availability of medical institutions have also to be considered. In addition, electronic data exchange before the patient's admission to a medical center must be fully supported in order to optimize the healthcare management process. The system architecture design we propose in this paper being based on the five main generic concepts: Actor, Resource, Location, Data and Telemedicine Task, is open enough to fulfill all these requirements and is able to support the various pervasive scenarios of telemedicine, whatever the actors who are involved, their available resources, their location, and the tasks they need to perform.

\section{REFERENCES}

[1] D. Saha and A. Mukherjee. "Pervasive Computing: A Paradigm for the 21st Century," Computer, vol. 36, 2003, pp. 25-31.

[2] N. Maglaveras et al. "The Citizen Health System (CHS): A Modular Medical Contact Center Providing Quality Telemedicine Services," IEEE Transactions on Information Technology in Biomedicine, 2005, pp. 353- 362

[3] J. Sachpazidis. “@HOME: A modular telemedicine system,” Mobile Computing in Medicine, Second Conference on Mobile Computing in Medicine, Workshop of the Project Group MoCoMed, GMDSFachbereich Medizinische Informatik \\&amp; GI-Fachausschuss 4.7, GI, 2002, pp. 87-95.

[4] R. Beuscart et al. "Homecare: The need for Cooperative Information Systems," MEDINFO 2004 IMIA, 2004, pp. 1343-1347.

[5] S.K. Yoo et al. "Prototype Design of Mobile Emergency Telemedicine System," Computational Science and Its Applications - ICCSA 2005, 2005, pp. 1028-1034.

[6] M. Eklund, T.R. Hansen, and J. Sprinkle. "Information Technology for Assisted Living at Home: building a wireless infrastructure for assisted living," IEEE.Engineering and Medicine in Biology Conference EMBC, Shanghai, China, 2005, pp. 3931-3934

[7] P. Rubel et al. "Towards Personal eHealth In Cardiology. Results from the EPI-MEDICS Telemedicine Project," J Electrocardiol, vol. 38 , 2005, pp. 1100-106

[8] J. Fayn et al. "Macro-Data: a New Approach for the Development of Tele-Expertise Applications," IEEE Computers in Cardiology, vol. 26, 1999, pp. 41-44.

[9] E. Christopoulou and A. Kameas. "Using Ontologies to Address Key Issues in Ubiquitous Computing Systems," Ambient Intelligence, 2004 pp. 13-24.

[10] "OWL Web Ontology Language Overview," http://www.w3.org/TR/owlfeatures/, 2004

[11] "Laboratory for Applied Ontology - DOLCE," http://www.loacnr.it/DOLCE.html.

[12] "Bfo - basic formal ontology - The Ontology Directory," http://ontologyonline.org/visualisation/c/Directory/Bfo+basic+formal+ontology/.

[13] "International Health Terminology Standards Development Organisation," http://snomed.org/.

[14] E. Christopoulou and A. Kameas. "GAS ontology: an ontology for collaboration among ubiquitous computing devices," Int. J. Hum.Comput. Stud., vol. 62, 2005, pp. 664-685.

[15] X. Wang et al. "Ontology based context modeling and reasoning using OWL," Pervasive Computing and Communications Workshops, 2004. Proceedings of the Second IEEE Annual Conference on, 2004, pp. 1822.

[16] H. Chen, T. Finin, and A. Joshi. "Semantic Web in the Context Broker Architecture," Proceedings of the Second IEEE International Conference on Pervasive Computing and Communications, IEEE Computer Society, 2004, pp. 277-286.

[17] T. Strang and C. Linnho Popien. "A Context Modeling Survey," The Sixth International Conference on Ubiquitous Computing, Workshop on Advanced Context Modelling, Reasoning and Management, Nottingham, England, 2004.

[18] "Ontology Definition Metamodel," www.omg.org/docs/ad/05-08-01.pdf, 2005.

[19] S. Ou et al. "A Model Driven Integration Architecture for OntologyBased Context Modelling and Context-Aware Application Development," Model Driven Architecture - Foundations and Applications, 2006, pp. 188-197.

[20] V. Bicer et al. "Artemis message exchange framework: semantic interoperability of exchanged messages in the healthcare domain," SIGMOD Rec., vol. 34, 2005, pp. 71-76.

[21] F. Paganelli and D. Giuli. "An Ontology-Based Context Model for Home Health Monitoring and Alerting in Chronic Patient Care Networks," Proceedings of the 21st International Conference on 
Advanced Information Networking and Applications Workshops Volume 02, IEEE Computer Society, 2007, pp. 838-845.

[22] P. Ganguly, M. Biomed, and N. Parameswaran. "Semantic Interoperability in Telemedicine through Ontology-Driven Services," Telemedicine and eHealth, vol. 11, 2005, pp. 405-412.

[23] E. Nageba, J. Fayn, and P. Rubel. "A Generic Task-Driven Multi-Agent Telemedicine System," Engineering in Medicine and Biology Society, 2007. EMBS 2007. 29th Annual International Conference of the IEEE, 2007, pp. 3733-3736

[24] E. Nageba, J. Fayn, and P. Rubel. "An Ontology-based Telemedicine Tasks Management System Architecture," 30th Annual International conference of the IEEE, Engineering in Medicine and Biology Society, Vancouver, BC Canada, 2008, pp. 1494-1497.

[25] "SPARQL Query Language for RDF," http://www.w3.org/TR/rdfsparql-query/, 2008.
[26] "SWRL: A Semantic Web Rule Language Combining OWL and RuleML," http://www.w3.org/Submission/SWRL/, 2004.

[27] "Jess, the Rule Engine for the Java Platform," http://www.jessrules.com/jess/index.shtml.

[28] "The Protégé Ontology Editor and Knowledge Acquisition System," http://protege.stanford.edw.

[29] "Racer Systems GmbH \& Co. KG," http://www.racer-systems.com/.

[30] G. De Luca, H. Suryapranata, J. P. Ottervanger, and E. M. Antman, "Time delay to treatment and mortality in primary angioplasty for acute myocardial infarction: every minute of delay counts," Circulation, vol 109, no. 10, pp. 1223-1225, March 2004.

[31] J. Fayn, L. Restier, B. Li, P. Chevalier, P. Rubel, "Assessment of the benefit of pervasive self-care ECG recording in arrhythmogenic patients," Eur. Heart J., vol. 27, no. Suppl. 1, pp. 140, 2006. 